\title{
A noncontact measurement technique for the specific heat and total hemispherical emissivity of undercooled refractory materials
}

\author{
Aaron J. Rulison a) and Won-Kyu Rhim \\ Jet Propulsion Laboratory, California Institute of Technology, 4800 Oak Grove Drive, Pasadena, \\ California 91109
}

(Received 20 September 1993; accepted for publication 13 December 1993)

\begin{abstract}
A noncontact measurement technique for the constant pressure specific heat $\left(c_{p l}\right)$ and the total hemispherical emissivity $\left(\epsilon_{T l}\right)$ of undercooled refractory materials is presented. In purely radiative cooling, a simple formula which relates the post-recalescence isotherm duration and the undercooling level to $c_{p l}$ is derived. This technique also allows us to measure $\epsilon_{T l}$ once $c_{p l}$ is known. The experiments were performed using the high-temperature high-vacuum electrostatic levitator at JPL in which 2-3 mm diameter metallic samples can be levitated, melted, and radiatively cooled in vacuum. The averaged specific heats and total hemispherical emissivities of $\mathrm{Zr}$ and $\mathrm{Ni}$ over the undercooled regions agree well with the results obtained by drop calorimetry: $c_{p l, \mathrm{av}}(\mathrm{Zr})=40.8 \pm 0.9 \mathrm{~J} / \mathrm{mol} \mathrm{K}, \epsilon_{T l, \mathrm{av}}(\mathrm{Zr})=0.28 \pm 0.01, c_{p l, \mathrm{av}}(\mathrm{Ni})=42.6 \pm 0.8 \mathrm{~J} / \mathrm{mol} \mathrm{K}$, and $\epsilon_{T l, \mathrm{av}}(\mathrm{Ni})=0.16 \pm 0.01$.
\end{abstract}

\section{INTRODUCTION}

The measurements of specific heat of a liquid $\left(c_{p l}\right)$ allow us to determine other thermodynamic parameters such as the enthalpy, entropy, and the Gibbs free energy. These quantities measured in the undercooled regions of various materials carry special implications for the studies of solidification processes and for the selection of useful metastable phases. ${ }^{1,2}$ However, data on $c_{p l}$ in the undercooled state are relatively scarce because deep undercooling is prohibited by heterogeneous nucleation which is primarily caused by contact with the container walls. In particular, due to their strong chemical reactivity with crucibles, $c_{p l}$ measurements of undercooled states of refractory materials had to wait until adequate containerless processing technologies became available.

There are several approaches one can take to measure $c_{p l}$ of undercooled melts: In drop calorimetry ${ }^{3,4}$ a melt undercooled to a known temperature is processed in a levitator, dropped into a calorimeter, and the heat released from the sample is quantified to determine the enthalpy, $H(T)$, as a function of undercooling temperature. $c_{p l}(T)$ is obtained by taking the gradient of $H(T)$ with respect to the temperature and dividing by the sample mass. Although this method is accurate, only one measurement can be made per sample.

In emulsion techniques, undercooling can be achieved by isolating the melts from container walls using appropriate oils which do not catalyze nucleation. ${ }^{5,6}$ Differential scanning calorimetry techniques are normally used to measure the heat transfer between the sample (the oil and melt combination) and the calorimeter. Emulsion techniques, however, are only available for relatively low-temperature materials such as $\mathrm{Hg}, \mathrm{In}, \mathrm{Sn}$, and $\mathrm{Bi}$. Barth et al. ${ }^{7}$ have recently applied a flux technique to measure $c_{p l}$ of nickel and iron. The use of the post-recalescence isotherm duration to measure the average specific heats of undercooled

\footnotetext{
a)NASA-NRC Resident Research Associate.
}

liquids is similar to the technique described in this paper except that our approach is purely containerless, i.e., no fluxes are required, and it also allows us to measure the total hemispherical emissivity. Further discussions on these points will be given in a later section.

In containerless processing techniques, an undercooled liquid sample is levitated using electromagnetic, acoustic, or electrostatic forces in order to isolate the sample from the container walls. The heat balance of a levitated liquid sample can be expressed by the following equation:

$m \frac{d}{d t}\left(c_{p l} T\right)=-\epsilon_{T} \sigma A\left(T^{4}-T_{s}^{4}\right)-h A\left(T-T_{s}\right)+Q_{\mathrm{in}}$,

where $m$ is the sample mass, $T$ is the sample temperature, $T_{s}$ is the temperature of the surroundings, $\epsilon_{T l}$ is the total hemispherical emissivity, $\sigma$ is the Stefan-Boltzmann constant, $A$ is the sample's surface area, $h$ is the heat-transfer coefficient which accounts for conductive and convective cooling in a gas, and $Q_{\text {in }}$ represents the heat input from external sources. All techniques for measuring $c_{p l}$ of a levitated sample must be based on Eq. (1).

Electromagnetic levitation intrinsically heats the sample at all times unless carried out in low gravity conditions, so, in Earth-based experiments, $Q_{\text {in }}$ is non-negligible and must be calculated or measured. Earth-based experiments also require cooling gases to lower the sample temperature. Thus the magnitude of the conductive and convective heat transfer expressed through the coefficient $h$ must be known. These effects combine to make measurement of $c_{p l}$ using electromagnetic levitators in Earth-based laboratories difficult.

Fecht and Johnson ${ }^{8}$ have proposed a technique which uses modulation of the heating power in electromagnetic levitation. The necessity, however, of measuring the total hemispherical emissivity $\epsilon_{T l}$ makes the power-modulation approach difficult.

In electrostatic levitation combined with radiant heating, $Q_{\text {in }}=0$ can be achieved in Eq. (1) simply by blocking the heating source. Since the processing environment can 
be high vacuum, the term including $h$ in Eq. (1) can be neglected. Thus the heat balance is purely radiative, and Eq. (1) can be reduced to

$$
m \frac{d}{d t}\left(c_{p l} T\right)=-\epsilon_{T l} \sigma A\left(T^{4}-T_{s}^{4}\right) .
$$

This equation is the basis for the measurements of $c_{p l}$ and $\epsilon_{T l}$ described in this paper.

\section{EXPERIMENTAL APPAIRATUS}

The experiments were performed using the hightemperature high-vacuum electrostatic levitator (HTHVESL) at the Jet Propulsion Laboratory. ${ }^{9}$ The HTHVESL uses feedback control to position samples between parallel plate electrodes. The sample and electrodes are contained in a stainless-steel vacuum chamber which is typically evacuated to $10^{-7}$ Torr. Samples $2.5 \mathrm{~mm}$ in diameter can be heated to about $2300 \mathrm{~K}$ using a focused 1 $\mathrm{kW}$ xenon arc lamp. Because electrostatic levitation does not intrinsically transfer heat to the sample, samples can be cooled to room temperature by extinguishing the arc lamp.

Sample radiance is recorded using a single-color optical pyrometer operating at $658 \mathrm{~nm}$ (filter width: $10 \mathrm{~nm}$ FWHM) and set to $2 \mathrm{kHz}$ bandwidth. The pyrometer views a spot on the sample about $1 \mathrm{~mm}$ in diameter. The radiance is converted to temperature using the method described by Hofmeister et al., ${ }^{10}$ wherein the spectral emissivity of the sample is assumed to be independent of temperature and equal to the value realized immediately after recalescence. The known melting temperature of the material is used to scale the data according to Planck's equation for the spectral radiance. The data acquisition and analysis are done automatically using a Macintosh-II computer.

\section{MEASUREMENT TECHNIQUE FOR SPECIFIC HEAT}

The present technique is based on the purely radiative cooling of an isolated spherical sample. Figure 1 shows a typical temperature versus time trace for a $40.0 \mathrm{mg}$ sample of zirconium. The section of the curve before point 1 is unusable because light from the arc lamp reflected from the sample surface into the pyrometer. The proper measurement of sample temperature begins at point 1 . The section after point 6 was obtained by blocking the pyrometer and is used for calibration purposes. At $t=0$, the sample had already reached a steady state temperature of $2195 \mathrm{~K}(67$ $\mathrm{K}$ above $T_{m}$ ). The sample was spherical and did not show any oscillation. At point 1, a shutter positioned in front of the arc lamp was closed to block the beam completely. In the absence of input energy, the sample cooled radiatively. At point 2, the sample had cooled to $T_{m}$. The sample remained liquid and continued to cool below $T_{m}$ to $1800 \mathrm{~K}$ at point 3 , at which time solidification started. Thus the sample was in the undercooled state during the time between points 2 and 3 . Solidification progressed rapidly and raised the sample's temperature to $T_{m}$ at point 4 due to recalescence. The sample approximately maintained $T_{m}$ until point 5 , after which its temperature fell again.

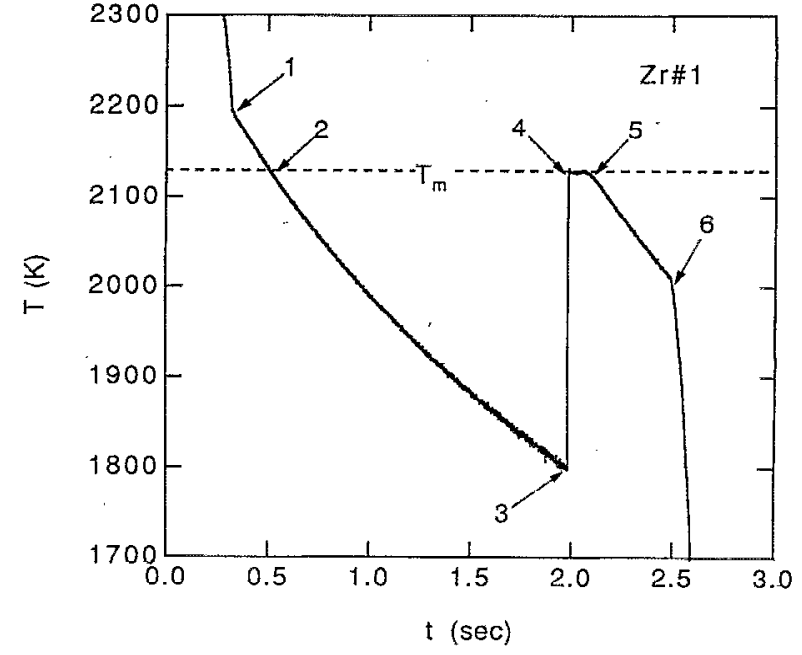

FIG. 1. A typical temperature $T$ vs time $t$ curve for a $40.7 \mathrm{mg}$ zirconium sphere (Zr No. 1) undergoing radiative cooling.

An energy balance can be written for the cooling process which occurred between any two points in the temperature versus time trace, assuming that the sample did not support large temperature gradients in its interior, i.e., the Biot number was small. Consider the balance of energy between points 2 and 5 in Fig. 1. Since the sample was completely molten at point 2 , its specific enthalpy here $h_{2}$ can be expressed by

$$
h_{2}=h_{f}+h_{5} \text {, }
$$

where $h_{f}$ is the specific enthalpy of fusion, and $h_{5}$ is the specific enthalpy at point 5 which is given by

$$
h_{5}=\int_{T_{\text {ref }}}^{T_{m}} c_{p s}(T) d T
$$

where $T_{\text {ref }}$ is an arbitrary reference temperature below $T_{m}$, and $c_{p s}$ is the specific heat capacity of the solid. If the temperature gradients in the sample are negligible, then the sample can be assumed to be completely solid at point 5 . Therefore, the difference in enthalpy between points 2 and 5 is the heat of fusion, $h_{f}$, and in the purely radiative cooling, it can be expressed by

$$
m h_{f}=-\int_{T_{2}}^{T_{3}} m c_{p l} d T+\int_{t_{4}}^{t_{5}} \epsilon_{T m} \sigma A\left(T_{m}^{4}-T_{s}^{4}\right) d t,
$$

where $\epsilon_{T m}$ is the total hemispherical emissivity in the isothermal region following recalescence. The heat emitted between points 3 and 4 is negligible because the time spent there is short compared to the time spent between points 2 and 5. Placing constant terms outside the integrals, Eq. (5) can be rewritten as

$$
m h_{f}=m \int_{T_{u}}^{T_{m}} c_{p l} d T+\sigma\left(T_{m}^{4}-T_{s}^{4}\right) \int_{t_{4}}^{t_{5}} \epsilon_{T m^{\prime}} A d t,
$$

where $T_{2}$ and $T_{3}$ have been replaced by $T_{m}$ and $T_{u}$, which are the melting temperature and the undercooled temperature just before the onset of solidification, respectively. The relationship between $T_{u}, t_{4}$, and $t_{5}$ can be measured 


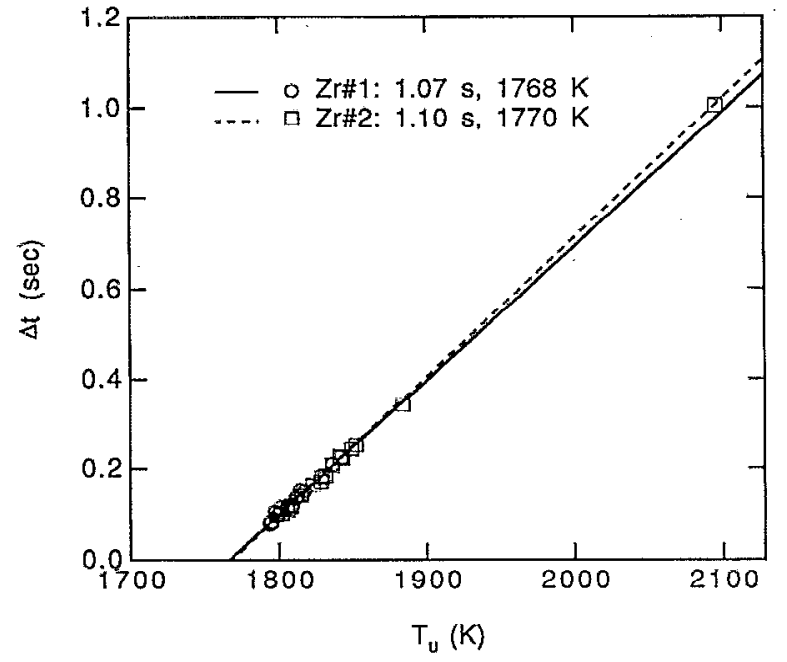

FIG. 2. Post-recalescence isotherm duration, $\Delta t\left(=t_{5}-t_{4}\right)$, vs undercooling temperature $T_{u}$ for $\mathrm{Zr}$. No. 1 (40.7 mg) and $\mathrm{Zr}$ No. 2 (40.0 mg). $\Delta t_{m}$ and $T_{\text {hyp }}$ are given in the legend.

experimentally using temperature versus time traces such as Fig. 1. These quantities can then be related to $c_{p l}$ and $\epsilon_{T m}$ using Eq. (6). It is important to note that the total hemispherical emissivity of the undercooled liquid, $\epsilon_{T}$, does not appear in Eq. (6), so it need not be measured. Rather, $c_{p l}$ depends on the total hemispherical emissivity at the melting temperature, $\epsilon_{T m}$. That is, accurate determination of $c_{p l}$ is in general hinged upon detailed knowledge of $\epsilon_{T m}$ which may or may not be available. However, a temperature-averaged $c_{p l}$ can be obtained without any knowledge of $\epsilon_{T m}$.

Consider the case where $t_{4}$ and $t_{5}$ are equal, i.e., the post-recalescence isotherm duration is zero. Then Eq. (6) becomes

$$
h_{f}=\int_{T_{u}}^{T_{m}} c_{p l} d T
$$

In this case $T_{u}$ is the temperature which marks the onset of hypercooling, $T_{\text {hyp }}$. The intersection of a plot of $\Delta t\left(=t_{5}-t_{4}\right)$ vs $T_{u}$ with the abscissa determines $T_{\text {hyp }}$ (see Fig. 2). Then, from Eq. (7), the average $c_{p l}$ of the undercooled liquid $c_{p l, \text { av }}$ is given by

$$
c_{p l, \mathrm{av}}=\frac{h_{f}}{T_{m}-T_{\mathrm{hyp}}} .
$$

Thus $c_{p l \text { av }}$ can be found without having any knowledge of the total hemispherical emissivity of the liquid or the solid. Equation (8) shows that the accuracy of $c_{p l \text { av }}$ depends directly on the accuracy of $h_{f}$, which must be measured by other means.

Now we consider the case where $T_{u}=T_{m}$, i.e., the liquid did not undercool. Equation (6) becomes

$$
m h_{f}=\sigma\left(T_{m}^{4}-T_{s}^{4}\right) \int_{t_{4}}^{t_{3}} A \epsilon_{T m} d t
$$

Assuming $A$ is constant, Eq. (9) may be used to find an average total hemispherical emissivity, $\epsilon_{T m, \text { av }}$, for the isotherm region, i.e.,

$$
\epsilon_{T m, \mathrm{av}}=\frac{m h_{f}}{\sigma\left(T_{m}^{4}-T_{s}^{4}\right) A \Delta t_{m}},
$$

where $\Delta t_{m}$ is determined from the intersection of a plot of $\Delta t\left(=t_{5}-t_{4}\right)$ vs $T_{u}$ with the ordinate (see Fig. 2). The sample density in the isotherm region must be known in order to calculate $A$.

If sufficient data on $\epsilon_{T m}$ is available to solve the second integral on the right-hand side of Eq. (6), then Eq. (6) can be used to calculate the temperature dependence of $c_{p l}$ in the undercooled region. Data on $\epsilon_{T m}$ required to calculate $c_{p l}\left(T_{u}\right)$ include its dependence on $T_{u}$ as well as its dependence on time after recalescence. Such data were not available for the present study.

Equation (2) shows that $c_{p l}\left(T_{u}\right)$ and $\epsilon_{T l}\left(T_{u}\right)$ determine the relationship between temperature versus time in the undercooled region. Thus, if either $c_{p l}\left(T_{u}\right)$ or $\epsilon_{T l}\left(T_{u}\right)$ is known, the other can be calculated from the cooling curve. Although in general the calculation must be made numerically, a simple analytical result can be derived if a plot of time versus $T^{-3}$ results in a straight line, in which case $\epsilon_{T} A / c_{p l}$ is constant. Then integration of Eq. (2) gives

$$
t_{f}-t_{0}=\frac{m c_{p l}}{3 \sigma \epsilon_{T t} A}\left(T_{f}^{-3}-T_{0}^{-3}\right),
$$

where 0 and $f$ refer to any two points in the undercooled region. If $c_{p l \text { av }}$ and $A$ are known, a straight line fitted to the data plotted as $t_{f}$ vs $T_{f}^{-3}$ can be used to determine an average total hemispherical emissivity of the undercooled liquid, $\epsilon_{T l, \mathrm{av}}$.

Since $c_{p l}\left(T_{u}\right)$ is determined by the distribution of points on the $\Delta t$ vs $T_{u}$ plot, the accuracy of $c_{p l}\left(T_{u}\right)$ will improve as the data points are distributed more widely over the temperature range between $T_{m}$ and $T_{\text {hyp }}$.

\section{EXPERIMENTAL PROCEDURES AND RESULTS}

\section{A. Zirconium}

Zirconium samples of $99.95 \%$ nominal purity were obtained from Teledyne Wah-Chang, Albany, Oregon, and prepared at Vanderbilt University by arc-melting in an argon atmosphere on a water-cooled plate to form them into approximate spheres. Experiments were performed on two zirconium samples, $\mathrm{Zr}$ No. 1 and $\mathrm{Zr}$ No. 2, whose masses were 40.7 and $40.0 \mathrm{mg}$, respectively. Plots of $\Delta t$ vs $T_{u}$ for both samples are shown in Fig. 2. There was some natural variation of $T_{u}$ from one undercooling experiment to the next, but the range was quite narrow. Therefore, in order to extend the range of $T_{u}$, a preliminary method of triggering nucleation was used. This consisted of bleeding in oxygen to form patches of an oxide layer on the sample's surface. Although successful in triggering nucleation over a wider range of undercooling temperatures than occurred naturally, this method is likely to have altered the total hemispherical emissivity of the sample, and is, therefore, far from ideal. But, the video records show that the surface 
area covered by the oxide layer patches was limited to a few percent of the sample's total area, so the results presented below are not affected greatly, although spikes in the radiance measurement were observed when oxide patches moved into the area on the pyrometer viewing area. Other methods for triggering nucleation are under development.

The temperature reading in the post-recalescence isotherm region did not remain exactly constant. For example, in one run it fell $7 \mathrm{~K}$ over $0.85 \mathrm{~s}$ isotherm duration, which is $0.33 \%$ of $T_{m}$. This may be due in part to the changing spectral emissivity and in part due to an actual surface temperature variation. If the total hemispherical emissivity changed while the actual surface temperature remained constant, then a $0.33 \%$ error would appear in the measured $\Delta t$. If the variation in the temperature reading was due to an actual surface temperature change then it must result in slower cooling than assumed. This effect could be reconciled by applying a correction factor which would result in a $1.3 \%$ decrease in the measured $\Delta t$. Any systematic error in $\Delta t$ cannot affect $c_{p l, a v}$ appreciably since it would not affect $T_{\text {hyp }}$ in Eq. (8), while it can affect $\epsilon_{T m \text {,av }}$ proportionately through $\Delta t_{m}$ in Eq. (10). In this study, however, no such corrections have been applied since the errors in $\Delta t$ are smaller than the experimental uncertainties associated with $h_{f}$ and $\rho_{m}$.

$\Delta t$ vs $T_{u}$ plots are shown in Fig. 2 . The data points are well represented by straight lines over the range of undercooling temperatures tested. Since $\Delta t_{m}$ and $T_{\text {hyp }}$ were not realized experimentally, it seems reasonable to determine them by extrapolating straight line fits to the data. The hypercooling temperatures for $\mathrm{Zr}$ No. 1 and $\mathrm{Zr}$ No. 2 were determined to be $1768 \pm 5$ and $1770 \pm 2 \mathrm{~K}$, respectively. Using Eq. (8) the values of the average specific heat capacities of the undercooled liquid $c_{p l, a v}$ of $\mathrm{Zr} \mathrm{No}$. 1, Zr. No. 2 are determined to be $40.7 \pm 0.9$ and $40.9 \pm 0.9 \mathrm{~J} / \mathrm{mol} \mathrm{K}$, assuming $h_{f}=14.652 \pm 0.32 \mathrm{~kJ} / \mathrm{mol} .{ }^{11}$ The value of $c_{p 1}\left(T_{m}\right)$ determined by Bonnel (1972) using drop calorimetry is $40.7 \pm 0.7 \mathrm{~J} / \mathrm{mol} \mathrm{K}$, which agrees well with the present result for the undercooled liquid. We emphasize that the accuracy of $c_{p l \text { av }}$ depends directly on the accuracy of $h_{f}$. Thus the measurement of $c_{p l, a v}$ may be refined if a more accurate value of $h_{f}$ becomes available.

Using Eq. (10) and the $\Delta t$ intercepts in Fig. 2, the values of the average total hemispherical emissivities at $T_{m}, \epsilon_{T m, \mathrm{av}}$, for $\mathrm{Zr}$ No. 1 and $\mathrm{Zr}$ No. 2 are determined to be $0.30 \pm 0.01$ and $0.29 \pm 0.01$, respectively. We have used $\rho_{m}=5920 \mathrm{~kg} / \mathrm{m}^{3}$ to compute the sample's surface area $A$. The density in the isotherm region was approximated by taking the average between the liquid and solid densities at $T_{m}$. The liquid and solid densities at $T_{m}$ were obtained from handbooks (liquid: Ref. 12; solid: Ref. 13). Note that the accuracy of $\epsilon_{T m, \text { av }}$ depends on the accuracy of $h_{f}$ and $\rho_{m}$, and may be refined as more accurate values become available.

Figure 3 shows the data of Fig. 1 plotted as $t$ vs $T^{-3}$. Using a best-fit straight line to the plot and Eq. (11), the value of the averaged total hemispherical emissivity of the undercooled liquid, $\epsilon_{T l, \mathrm{av}}$, for $\mathrm{Zr}$ No. 1 is determined to be

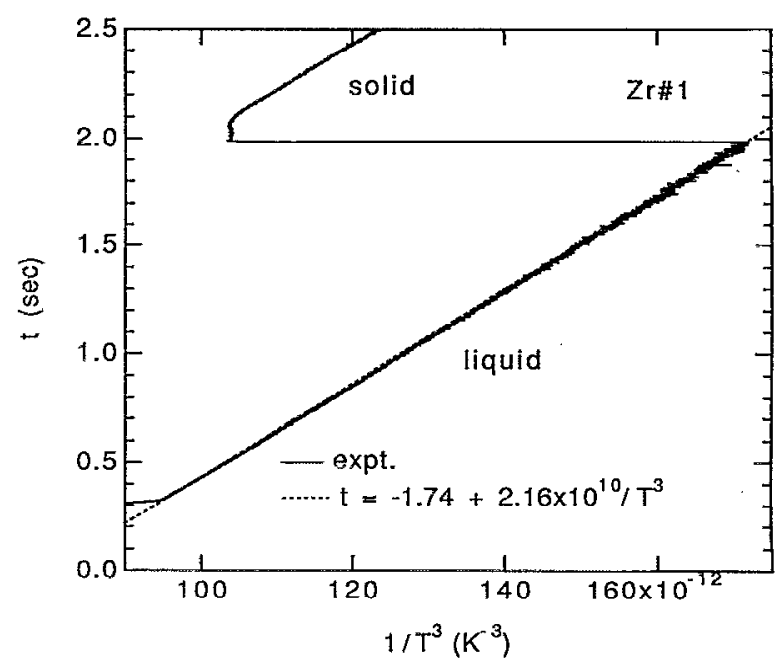

FIG. 3. A typical $t$ vs $T^{-3}$ plot for $\mathrm{Zr}$ No. 1 (40.7 mg). A best-fit straight line is superimposed on the experimental data.

$0.27 \pm 0.01$, assuming $\rho_{l}=5600 \mathrm{~kg} / \mathrm{m}^{3}$ to compute $A .^{12}$ The second sample, $\mathrm{Zr}$ No. 2 , resulted in $0.28 \pm 0.01$. The accuracy of $\epsilon_{T l, \text { av }}$ depends on the accuracy of available values of $h_{f}$ and $\rho_{l}$. There appear to be no published data for the total hemispherical emissivity of zirconium in any form. The spectral emissivities at $650 \mathrm{~nm}$ for liquid and solid zirconium are reported to be 0.32 and 0.30 , respectively. ${ }^{12}$ The measured value of $\epsilon_{T m, \text { av }}$ may have been affected by a roughening of the surface observed during solidification.

\section{B. Nickel}

A typical temperature versus time trace for a $19.4 \mathrm{mg}$ sample of nickel of $99.99 \%$ nominal purity (Electronic Space Products, Int'1) is shown in Fig. 4. The pattern of superheating, undercooling, and recalescence is similar to that of zirconium as shown in Fig. 1, except the time re-

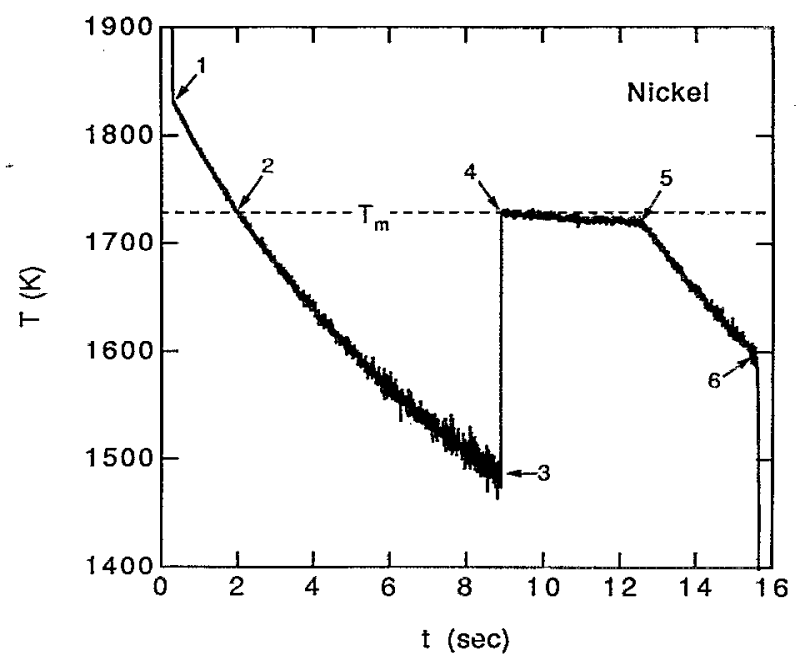

FIG. 4. A typical temperature vs time curve for a $19.4 \mathrm{mg}$ nickel sphere undergoing radiative cooling. 


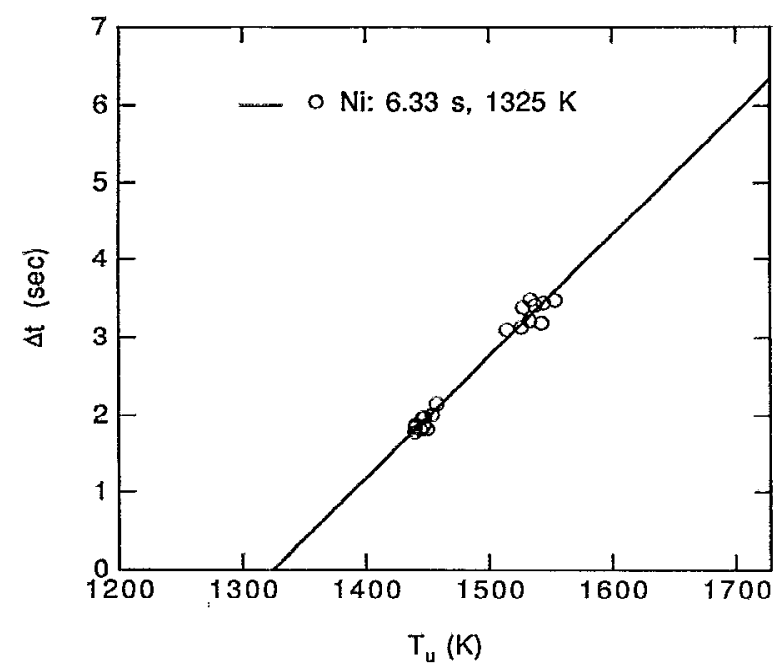

FIG. 5. $\Delta t\left(=t_{5}-t_{4}\right)$ vs $T_{u}$ for the nickel sample (19.4 mg). $T_{\text {hyp }}$ and $\Delta t_{m}$ are given in the legend.

quired to cool the sample is greater because the lower operating temperature led to lower radiative heat fluxes.

As was the case for zirconium, the temperature reading in the post-recalescence isotherm region did not remain exactly constant. For example, in one run it fell $8 \mathrm{~K}$ over $3.65 \mathrm{~s}$ isotherm duration, which is $0.46 \%$ of $T_{m}$. For the similar reasons given for zirconium, no corrections have been applied.

Figure 5 shows the $\Delta t$ vs $T_{u}$ data. Although the choice of a straight line to fit the data is not as obvious in this case as it was for zirconium, a best-fit straight line has been used to find $\Delta t_{m}$ and $T_{\text {hyp }}$. The hypercooling temperature was determined to be $1325 \pm 4 \mathrm{~K}$. Using Eq. (8) the value of the average specific heat capacity, $c_{p l, a v}$, of the nickel sample is determined to be $42.6 \pm 0.4 \mathrm{~J} / \mathrm{mol} \mathrm{K}$, assuming $h_{f}=17.15 \mathrm{~kJ} / \mathrm{mol}^{14} \quad c_{p l}\left(T_{m}\right)$ is reported to be 38.49 $\mathrm{J} / \mathrm{mol} \mathrm{K},{ }^{14}$ which is close to the present result for the undercooled liquid. Barth et al. ${ }^{7}$ found $c_{p l}\left(T_{m}\right)=41 \pm 2$ and $43 \pm 2 \mathrm{~J} / \mathrm{mol} \mathrm{K}$ using drop calorimetry and flux techniques, respectively, which are also close to the present result. We emphasize once again that the accuracy of $c_{p l, \mathrm{av}}$ depends directly on the accuracy of $h_{f}$. Thus the measurement of $c_{p l, \mathrm{av}}$ may be refined if a more accurate value of $h_{f}$ becomes available.

Using Eq. (10) and the $\Delta t$ intercepts, the value of the average total hemispherical emissivity at $T_{m}, \epsilon_{T m, \text { av }}$, for the nickel sample is determined to be $0.21 \pm 0.01$, assuming $\rho_{m}=8100 \mathrm{~kg} / \mathrm{m}^{3}$. The density during the isotherm region, $\rho_{m}$, is obtained by averaging the liquid and solid densities at $T_{m}$ (liquid: Ref. 12; solid: Ref. 15). The accuracy of $\epsilon_{T m, \mathrm{av}}$ depends on the accuracy of $h_{f}$ and $\rho_{m}$ and may be refined in the future.

Figure 6 shows the data of Fig. 4 plotted as $t$ vs $T^{-3}$. Using a best-fit straight line to the $t$ vs $T^{-3}$ plot and Eq. (11), the value of the averaged total hemispherical emissivity of the undercooled liquid, $\epsilon_{T l, a v}$, for the nickel sample is determined to be $0.16 \pm 0.01$, assuming $\rho_{l}=7900$ $\mathrm{kg} / \mathrm{m}^{3}$ to compute $A .^{12}$ The value of $\epsilon_{T l \text {,av }}$ is significantly

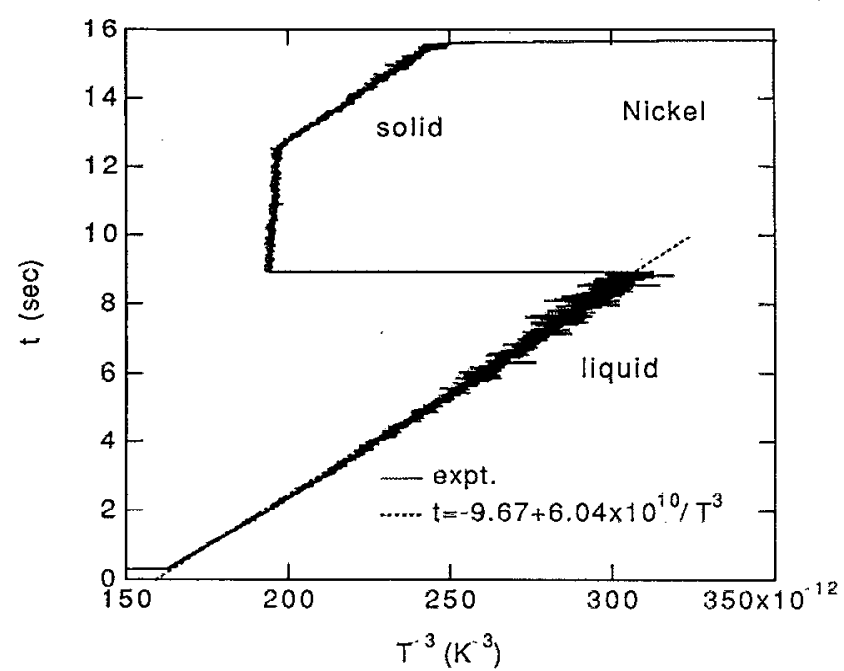

FIG. 6. A typical time vs $T^{-3}$ plot for the nickel sample (19.4 mg). A best-fit straight line is superimposed on the experimental data.

less than $\epsilon_{T m, \text { av }}$. This may be due in part to a roughening of the surface that was observed during solidification. Again, the accuracy of $\epsilon_{l, \mathrm{av}}$ depends on the accuracy of $h_{f}$ and $\rho_{l}$ available in the literature.

The total hemispherical emissivity of solid nickel is reported to be 0.12 at $773 \mathrm{~K}$ and 0.19 at $1273 \mathrm{~K} .{ }^{12}$ Extrapolating to $T_{m}$, a value of 0.25 is obtained, which is in rough agreement with the present value of $\epsilon_{T m, a v}=0.21$.

The thermophysical properties of zirconium and nickel are summarized in Table I.

\section{DISCUSSION}

The present measurement technique is similar to the flux technique of Barth et al., ${ }^{7}$ in that both use the postrecalescence isotherm duration to find $T_{\text {hyp }}$, which, in turn, determines the average specific heat of undercooled melts. The present technique, however, is truly containerless, which brings about two main advantages. First, refractory and reactive materials for which no fluxes are

TABLE I. Thermophysical properties of $\mathrm{Zr}$ and $\mathrm{Ni}$.

\begin{tabular}{lcc}
\hline \hline Property material & $\mathrm{Zr}$ & $\mathrm{Ni}$ \\
\hline$c_{p l}(\mathrm{~J} / \mathrm{mol} \mathrm{K})$ & $40.8 \pm 0.9,^{\mathrm{a}} 40.7^{\mathrm{b}}$ & $42.6 \pm 0.4,^{\mathrm{a}} 39.3,,^{\mathrm{b}} 38.5,{ }^{\mathrm{e}} 39.3^{\mathrm{f}}$ \\
$\epsilon_{T m, \mathrm{av}}$ & $0.30 \pm 0.01^{\mathrm{h}}$ & $0.21 \pm 0.01^{\mathrm{h}}$ \\
$\epsilon_{T l, \mathrm{av}}$ & $0.28 \pm 0.01^{\mathrm{h}}$ & $0.16 \pm 0.01^{\mathrm{h}}$ \\
$T_{\text {hyp }}(\mathrm{K})$ & $1769 \pm 5^{\mathrm{h}}$ & $1325 \pm 4,{ }^{\mathrm{h}} 1318 \pm 20^{\mathrm{i}}$ \\
$\rho_{s}\left(T_{m}\right)\left(\mathrm{kg} / \mathrm{m}^{3}\right)$ & $6240^{\mathrm{c}}$ & $8400^{\mathrm{g}}$ \\
$\rho_{l}\left(T_{m}\right)\left(\mathrm{kg} / \mathrm{m}^{3}\right)$ & $5600^{\mathrm{d}}$ & $7900^{\mathrm{e}}$ \\
$h_{f}(\mathrm{~kJ} / \mathrm{mol})$ & $14.652 \pm 0.32^{\mathrm{b}}$ & $17.15^{\mathrm{c}}$ \\
$T_{m}(\mathrm{~K})$ & $2128^{\mathrm{d}}$ & $1728^{\mathrm{d}}$ \\
\hline \hline
\end{tabular}

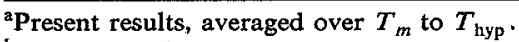

${ }^{b}$ Bonnell (Ref. 11) at $T_{m}$.

"Shaffer (Ref. 13).

${ }^{d}$ Weast and Astle (Ref. 12).

'Iida and Guthrie (Ref. 14) at $T_{m}$.

${ }^{f}$ Barth et al. (Ref. 7), averaged over $T_{m}$ to $T_{\text {hyp }}$

Brandes (Ref. 15).

hresent results.

${ }^{i}$ Barth et al. (Ref. 7), 
available can be processed. Second, the cooling process is purely radiative, i.e., it is unaffected by fluxes and gases. Therefore, the total hemispherical emissivities of the undercooled liquid and the solid after recalescence can also be determined.

Metallic and ceramic alloys can be superheated and undercooled as well as pure metals. Phase transformation processes in alloys are more complicated than in pure materials and they depend on the details of their phase diagrams. For example, the post-recalescence temperature of a Ag-Cu eutectic alloy is a function not only of the alloy's composition but of the undercooling temperature. ${ }^{16} \mathrm{Ob}$ taining the averaged specific heat of the undercooled liquid alloys should still be straightforward utilizing the interception of the $\Delta t-T_{u}$ plot with the $T_{u}$ axis to determine the hypercooling temperature. Obtaining the temperature dependence of the specific heat, however, will require more analysis because of the complexities involved in alloy solidification processes.

We have introduced in this paper a noncontact technique of measuring the specific heat and the total hemispherical emissivity of undercooled states of pure metals. The noncontact approach allowed us to achieve undercooled states of zirconium and nickel which are known to be highly reactive with most crucibles. Furthermore, the noncontact approach allowed us to measure both specific heat and total hemispherical emissivity. The technique introduced in this paper has important implications for the study of solidification processes; in particular, for the selection of various metastable states which have important engineering applications. Studies on more complex materials such as metallic alloys, semiconductors, and glasses are in progress and will be published elsewhere.

\section{ACKNOWLEDGMENTS}

We gratefully acknowledge Professor R. Hauge of Rice University for providing us with a preprint of his extensive survey on liquid metal property data. We thank Dr. Eugene Trinh and Dr. K. Ohsaka of the Jet Propulsion Laboratory for valuable discussions and critical comments on the manuscript, Professor F. G. Shi of the University of California, Irvine, for bringing an important paper by Barth et al., to our attention, Mr. Craig Morton of Vanderbilt University for providing the zirconium samples, $\mathrm{Mr}$. Kelly Perry for developing the data analysis software, and Dr. Kin Fung Man, Mr. Daniel Barber, and Mr. Robert McMillan for their generous help throughout the experiments. This work was carried out at the Jet Propulsion Laboratory, California Institute of Technology, under contract with the National Aeronautics and Space Administration.

${ }^{1}$ D. Turnbull, J. Appl. Phys. 21, 1022 (1950).

${ }^{2}$ E. Schleip, D. M. Herlach, and B. Feuerbacher, Europhys. Lett. 11, 751 (1990).

${ }^{3}$ R. T. Grimley, Ph.D. thesis, University of Wisconsin, Madison, 1958.

${ }^{4}$ K. Ohsaka, J. R. Gatewood, and E. H. Trinh, Scr. Metall. Mater. 25, 1459 (1991).

${ }^{5}$ H. S. Chen and D. Turnbull, Acta Metall. 16, 369 (1968).

${ }^{6}$ J. H. Perepezko and J. S. Paik, J. Non-Cryst. Solids 61\&62, 113 (1984).

${ }^{7}$ M. Barth, F. Joo, B. Wei, and D. M. Herlach, J. Non-Cryst. Solids 156-158, 398 (1993).

${ }^{8}$ H. J. Fecht and W. L. Johnson, Rev. Sci. Instrum. 62, 1299 (1991).

${ }^{9}$ W. K. Rhim, S. K. Chung, D. Barber, K. F. Man, G. Gutt, A. Rulison, and R. E. Spjut, Rev. Sci. Instrum. 64, 2961 (1993).

${ }^{10}$ W. H. Hofmeister, R. J. Bayuzick, and M. B. Robinson, Rev. Sci. Instrum. 61, 2220 (1990).

${ }^{11}$ Bonnell, Ph.D. thesis, Rice University, 1972.

${ }^{12}$ CRC Handbook of Chemistry and Physics, $62 \mathrm{nd}$ ed., edited by R. C. Weast and M. J. Astle (CRC, Boca Raton, FL, 1981).

${ }^{13}$ P. T. B. Shaffer, Plenum Press Handbooks of High Temperature Materials No. 1, Materials Index (Plenum, Ncw York, 1964).

${ }^{14}$ T. Iida and R. I. L. Guthrie, The Physical Properties of Liquid Metals (Clarendon, Oxford, 1988).

${ }^{15}$ Smithells Metals Reference Book, 6th ed., ediled by E. A. Brandes (Butterworths, London, 1983).

${ }^{16} \mathrm{~K}$. Ohsaka and E. H. Trinh, Scr. Metall. Mater. 29, 845 (1993). 